\title{
The role of early invasive, selective invasive, or conservative strategies in acute coronary syndromes
}

\section{Jean Vorster and Pravin Manga}

Department of Medicine, Division of Cardiology, Johannesburg Hospital and University of Witwatersrand

Address for correspondence:

DrJ.Vorster

Department of Cardiology

PO Box 148|

Houghton

2041

\section{Email:}

Pravin.Manga@wits.ac.za

\section{ABSTRACT Acute Coronary Syndrome (ACS)} comprises three related disorders. In ST-elevation myocardial infarction (STEMI) rapid restoration of flow in the infarct-related artery (via pharmacological or mechanical means) is of paramount importance to minimize necrosis. In patients presenting with Unstable Angina / Non-ST-segment myocardial infarction (UA/ NSTEMI) the correct choice of the initial treatment strategy (early invasive vs. selective invasive vs. conservative) is imperative in assuring optimal patient outcomes. Various risk prediction models can assist in the decision making process in an individual patient. The timing of angiography and revascularization in patients selected for the early invasive strategy are important factors in determining the long-term outcome in this patient population subset. SAHeart 2008; 5:58-64

\section{INTRODUCTION}

Acute Coronary Syndrome (ACS) comprises three related disorders: ST-elevation myocardial infarction (STEMI), unstable angina (UA), and non-ST-elevation myocardial infarction (NSTEMI).The initial management of STEMI differs considerably from that of UA and NSTEMI, which is generally considered as one clinical entity (UA/NSTEMI). STEMI, which is responsible for $25 \%$ of ACS cases, occurs as a result of total occlusion of an epicardial coronary artery by an intra-luminal thrombus after rupture or erosion of an unstable atherosclerotic plaque. Rapid restoration of flow in the infarct-related coronary artery is of paramount importance to limit the infarct size. Thus primary $\mathrm{PCl}$ has become the standard of care in appropriate settings and definitive goals have been set in the form of Door-to-Balloon time of less than 90 minutes for primary $\mathrm{PCl}$ and Door-to-Needle time of less than 60 minutes for fibrinolytic therapy. ${ }^{(1)}$ The South African situation is similar to that in many parts of the world, in that only a small percentage of patients presenting with a STEMI will be treated with primary $\mathrm{PCl}$. The major reason is the limited availability of facilities in South Africa that can offer primary $\mathrm{PCl}$. Fibrinolysis is therefore still the primary form of reperfusion therapy in the majority of patients. Thus in the South African context, issues of improvement of fibrinolytic therapy by adjunctive $\mathrm{PCl}$, rescue $\mathrm{PCl}$ and late $\mathrm{PCl}$ are important. However, this is the subject matter of another review in this journal.

UA/NSTEMI differs from STEMI in that it is associated with a subtotal occlusion of the epicardial coronary artery, with some, albeit insufficient, flow past the unstable plaque and intra-luminal thrombus. Current guidelines suggest that patients presenting with UA/NSTEMI who have persistent or recurrent angina with / without ST-segment changes ( $\geq 2 \mathrm{~mm})$ or deep negative T-wave inversion despite optimal medical therapy; patients with signs of heart failure or hemodynamic instability; or life-threatening arrhythmias should be referred for urgent angiography / revascularization. (I) These patients, however, comprise only $2-15 \%$ of the patients admitted to hospital with UA/NSTEMI. It is the management of the remaining large group of ACS patients that is up for debate.

Two different treatment strategies may be followed after initial medical stabilization of UA/NSTEMI patients who do not meet the criteria for urgent angiography / revascularization. The first is an early invasive (routine) strategy of coronary angiography and, if indicated, revascularization. The second is a conservative (selective invasive) 
strategy in which patients, after appropriate medical stabilization, undergo coronary angiography and revascularization only if there is evidence of recurrent ischemia, e.g. recurrent infarction, angina at rest, dynamic ST-segment changes on ECG or inducible ischemia. Two alternatives for the invasive approach have emerged: early ("immediate") or deferred angiography, i.e. after a 12 to 48-hour window from symptom onset.

The choice of strategies has led to many debates at conferences and in the literature and there is widespread variation in procedure use among individual clinicians, institutions and countries. ${ }^{(2)}$ Proponents of the early invasive strategy argue that the early determination of coronary anatomy can be used to tailor therapy, avoid lengthy hospital stay and prevent further ischemic events. Proponents of the conservative strategy argue that medical therapy can stabilize patients and that noninvasive testing can identify patients at risk of future events and thus identify patients who would most benefit from invasive intervention. Thereby, the costs and complications of invasive procedures can be minimized by using these modalities selectively. The last argument is particularly relevant to our own practice environment in South Africa, where resources are limited, especially in the public sector. Over the last decade many randomized trials and large scale registries have been published on this topic. The evidence for the relative benefit or harm of these two approaches, as well as patient selection for the respective strategies and optimal timing for coronary angiography is the subject of this review.

\section{CHOICE OF STRATEGY}

A meta-analysis of seven randomized trials (including early studies prior to the widespread use of stents and multi-drug adjunctive therapy) conducted by Metha et al., consisting of 9212 patients, evaluated the benefits and risks of early invasive vs. selective invasive strategies in UA/NSTEMI over a mean follow-up period of 17 months. ${ }^{(3)}$ Overall, death or MI was reduced from $14.4 \%$ in the selective invasive group to $12.2 \%$ in the early invasive group (odds ratio [OR], 0.82; $p<0.001$ ). This was largely driven by a significant reduction in $\mathrm{MI}(9.4 \%$ vs. $7.3 \%$; OR, 0.75; $p<0.001$ ). There was a non-significant trend toward a lower mortality with the early invasive strategy (6.0\% vs. 5.5\%; OR, 0.92; $p<0.33)$
The early invasive strategy was, however, associated with a significantly higher early mortality $1.8 \%$ vs. I. $1 \%$ for early vs. selective invasive, respectively $(\mathrm{OR}$ I.60; $\mathrm{p}<0.007$ ) and the composite of death or $\mathrm{MI}$ (5.2\% vs. 3.8\%; OR I.36; $p<0.002$ ). The beneficial effect of the early invasive strategy was actually achieved from hospital discharge to the end of follow-up (17 months) where the early invasive strategy was associated with fewer subsequent deaths (3.8\% vs. 4.9\%; $p<0.01$ ) and the composite of death or $\mathrm{MI}(7.4 \% \mathrm{vs} .11 .0 \% ; \mathrm{p}<0.00 \mathrm{I})$. At the end of follow-up, there was also a 33\% reduction in severe angina ( 1 1.2\% vs. $14.0 \%$ ) and a $34 \%$ reduction in rehospitalization (32.5\% vs. $41.3 \%$ ) in patients who were stratified to the early invasive strategy. ${ }^{(3)}$

A clue to risk stratification was provided by the biomarker data. Higher risk patients with elevated cardiac biomarkers at baseline benefited more from early invasive intervention, with no significant benefit observed in lower-risk patients with negative baseline biomarker levels.

The ICTUS trial(4) was not included in the meta-analysis, as its results were published after the publication of the Mehta et al. meta-analysis. The results of the ICTUS trial challenged the paradigm of a superior outcome of an early invasive strategy. In this trial | 200 high-risk ACS patients (troponin positive) were randomly assigned to either an early invasive (angiography within 24 to 48 hours) or a selectively invasive strategy. All patients received optimal medical therapy that included aspirin, clopidogrel, enoxaparin, abciximab during $\mathrm{PCl}$ and high dose statins. In this trial there was no difference in the primary composite end-point of death, MI, or rehospitalization for angina at I year $22.7 \%$ vs. $21.2 \%$; $=0.33$ ) with an early vs. selective invasive strategy. Mortality at one year was identical at 2.5\%. In keeping with previous studies, early intervention was associated with a significant early hazard of $\mathrm{MI}$ (I5.0\% vs. 10.0\%; RR I.5 p <0.005). Furthermore, the point estimate for the relative risk of the composite primary end point of death, $\mathrm{Ml}$ or rehospitalization for angina favoured patients assigned to the selective invasive group for the entire follow-up period of I year.The long-term follow-up data of the ICTUS study cohort was recently published. ${ }^{(5)}$ The primary composite endpoint was 30\% in the early invasive group and $26 \%$ in the selective invasive group $(p=0.09)$. No difference in overall cardiovascular mortality was noted between early invasive and selective invasive strategies ( $4.5 \%$ vs $5.0 \% p=0.97$ ). 
The discrepancy between the ICTUS study and the results of the Mehta meta-analysis could, in part, be attributed to the relatively high rates of revascularization in the two study groups of the ICTUS trial $76 \%$ in the invasive and $40 \%$ in the selective invasive group).(4) Another factor could be that all patients in the ICTUS study received intensive medical therapy.

One of the weaknesses cited of the meta-analysis by Mehta et al. was that many of the trials included in the analysis were not contemporary to current clinical practice. In four of the trials, namely TIMI-3B, ${ }^{(6)}$ VANQWISH. ${ }^{(7)}$ MATE, ${ }^{(8)}$ and FRISC-2,(9) the use of stents and GP IIb/ Illa inhibitors was low or non-existent. However, a recent review of the more contemporary trials by the Cochrane collaboration confirmed the initial observations reported by Mehta et al. In this systematic review, which included the ICTUS trial, mortality during initial hospitalization showed a trend to hazard with an early invasive strategy (RR, I.59; 95\% Cl, 0.96-2.64).(10) However, late mortality, at 2-5 year follow-up was significantly decreased by $25 \%(R R 0.75 ; p=0.09)$ with an early invasive strategy as compared to the conservative strategy. It must be noted, however, that this analysis was done prior to the publication of the 3-year data of the ICTUS study, which found no difference in mortality at 3 years follow-up. ${ }^{(5)}$ The incidence of refractory angina was also significantly reduced by $33 \%(\mathrm{RR} 0.67 \mathrm{Cl}, 0.55-0.83)$ with an invasive strategy at 6-12 months. An early invasive strategy also further reduced the rehospitalization rates, with a relative risk of $0.67(95 \% \mathrm{Cl}$, $0.61-0.74)$ at $6-12$ months. The early invasive strategy, however, was associated with a two-fold increase in the relative risk of peri-procedural $\mathrm{Ml}$ and a 1.7-fold increase in the relative risk of bleeding.

The most recent meta-analysis published on the subject included 7 studies comprising 8375 patients. ${ }^{(1)}$ These studies reflect contemporary practice in that all patients received aspirin, unfractionated or low molecular weight heparin. Glycoprotein Ilb/llla inhibitors were available during $\mathrm{PCl}$ in 6 of the 7 trials and thienopyridines were used as an adjunct to $\mathrm{PCl}$ in all trials. For the primary outcome of all-cause mortality at a mean follow-up of 2 years there was a significant risk reduction for all-cause mortality from $6.5 \%$ for the conservative strategy to $4.9 \%$ for an early invasive strategy $(R R, 0.75 ; p=0.00 I)$, without an excess of early death (Figure I). The analysis of all-cause mortality showed no heterogeneity between studies. Interestingly the
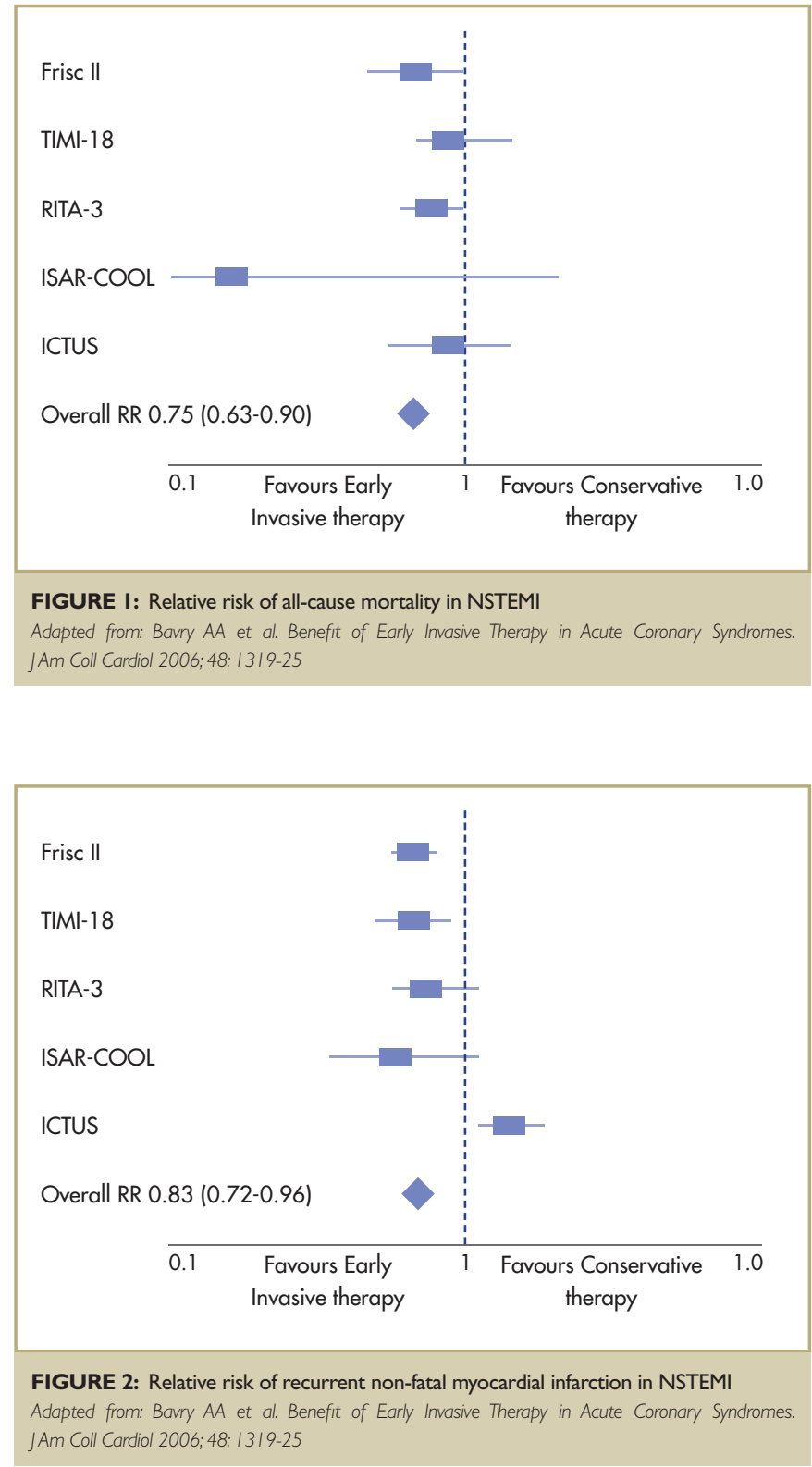

analysis of mortality demonstrated that with longer follow-up, the mortality benefit increased. Thus the risk reduction at I month was 18\%, at 6 months 17\%, at 12 months $20 \%$ and at 2 years $25 \%$. Furthermore, the incidence of non-fatal MI at 2-year follow-up was lower with the early invasive versus the conservative approach (7.6\% vs. $9.1 \% \mathrm{p}=0.012$ ) again without an excess of early non-fatal MI (Figure 2). Long-term mortality reduction has further been confirmed in the 5-year follow-up data of the RITA-3(12) trial and the 2-year followup data of the FRISC-2 trial.(9) However, it must be noted that the longterm follow-up at 3 years of the ICTUS study showed no mortality difference between the two treatment strategies. ${ }^{(5)}$ 
Based on the above evidence, it is reasonable to conclude that in patients with UA/NSTEMI, a routine early invasive strategy is preferable to a selective invasive strategy in reducing major adverse cardiovascular events as well as severe angina and rehospitalization. However, it appears that the major benefit of an early invasive strategy resides in reducing cardiovascular events over the long term with the risk that this strategy may be associated with an early hazard of myocardial infarction. Therefore, future strategies should focus on ways of reducing the early hazard and enhancing the later benefits of a routine invasive strategy. ${ }^{(3)}$

It is also important to note that in almost all the randomized trials, a significant proportion of patients in the conservative arm eventually underwent revascularization ("crossover"), such that the benefit of revascularization may have been underestimated. ${ }^{(10)}$

\section{TIMING OF INVASIVENESS}

With the exception of indications for emergency / urgent angiography and revascularization, controversy remains as to the optimal timing between hospital admission, initiation of medical therapy, and the invasive evaluation.

Support for immediate angiography comes from the ISAR-COOL trial. ${ }^{(13)}$ In this trial, 410 consecutive, high-risk NSTEMI patients were treated with intensive medical therapy; including aspirin, heparin, clopidogrel (600mg loading dose), and the Glycoprotein Ilb/llla inhibitor, tirofiban. Patients were randomized to immediate angiography (on average 2.4 hours after admission) or delayed angiography after a prolonged "cooling off" period before catheterization (86 hours after admission and medical therapy). Patients randomized to immediate $\mathrm{PCl}$ had a lower incidence of death or $\mathrm{MI}$ at 30 days than patients randomized to deferred $\mathrm{PCl}(5.9 \%$ vs. I I.6\% p $=0.04)$. Likewise, no early hazard was observed in the TACTICS-TIMI- I 8 study wherein patients were treated with upstream Glycoprotein IIb/Illa inhibitor and where the mean delay to $\mathrm{PCl}$ was 22 hours. ${ }^{(14)}$
At variance with these findings, are the results of the ICTUS trial, which showed that early routine invasive strategy within 48 hours after randomization was associated with an excess of MI.(4) Expedited catheterization was also associated with worse outcome in the FRISC- $2^{(9)}$ study as well as in the CRUSADE registry. ${ }^{(15)}$

Accordingly, current data does not mandate a systematic approach of immediate angiography in UANNSTEMI patients stabilized with contemporary medical therapy. Both the $\mathrm{ESC}^{(1)}$ and $\mathrm{ACC} / \mathrm{AHA}^{(16)}$ guidelines for the management of patients with UA/NSTEMI suggest that angiography / revascularization can be performed safely within 4 to 72 hours of admission provided the patient is stabilized with optimal medical therapy.

\section{SELECTION OFTHE INITIALTREATMENT STRATEGY BASED ON RISK FACTORS}

The primary objective in selecting a treatment strategy in UA/NSTEMI is to yield the best long-term clinical outcome. In the individual patient the risk or hazard of an early invasive angiography / revascularization should be weighed against the long-term benefit accrued from such a strategy. Risk stratification is an integral component of managing patients presenting with UA/NSTEMI.The goal of risk stratification is to identify patients with a high likelihood of an early complicated outcome who are at risk of recurrent coronary events or premature death and to offer such patients the benefit of early angiography and revascularization. Patients presenting with persistent or recurrent ischemia, hemodynamic or electrical instability, or heart failure have a particularly high risk for major adverse cardiovascular events, and therefore benefit from urgent angiography with revascularization (PCl or $\mathrm{CABG}$ ).

The current guidelines ${ }^{(1,16)}$ recommend the use of several parameters for risk stratification for patients presenting with UA/NSTEMI, e.g. the TIMI (17) risk score (Table I) or GRACE(18) risk score (Table 2). Patients presenting with moderate to high risk features according to one of these scoring systems also attain significant benefit from an early / 
TREATMENT

STRATEGIES IN ACS

TABLE I: TIMI Risk Score for UA/NSTEMI(17)

Age $\geq 65$ years

$\geq C A D$ risk factors

Known CAD ( $>50 \%$ stenosis)

Prior Aspirin

2 Anginal episodes in the preceding 24 hours

ST deviation $\geq 0.5 \mathrm{~mm}$

Raised cardiac biomarkers

\begin{tabular}{|c|c|}
\hline TIMI Risk & Score \\
\hline Low & $0-2$ \\
\hline Intermediate & $3-4$ \\
\hline High & $5-7$ \\
\hline
\end{tabular}

TABLE 3: Characteristics associated with intermediate to high risk of an adverse cardiac event in patients with UA/NSTEMI(i)

Elevated troponin levels

Dynamic ST- or T-wave changes (symptomatic or silent)

Diabetes mellitus

Renal dysfunction (GFR < $60 \mathrm{ml} / \mathrm{min} / 1.73 \mathrm{~m}^{2}$ )

Reduced left ventricular function $(E F<40 \%)$

Early post-infarction angina

Prior $\mathrm{Ml}$

$\mathrm{PCl}$ within 6 months

Prior $\mathrm{CABC}$

Intermediate to high TIMI / GRACE risk score

routine invasive strategy. The recently published guidelines ${ }^{(1)}$ emphasize that patients with characteristics as listed in Table 3, be classified as intermediate to high risk for major adverse cardiovascular events in the foreseeable future and should be submitted to an early invasive strategy.

Coronary angiography, by its very nature, risk stratifies patients. Not only does it define the degree and extent of coronary artery disease and thus the risk for recurrent events or mortality; but it is also a way of determining left ventricular function which in itself is a strong predictor of outcome in a wide spectrum of patients. Angiography is therefore clearly an important tool in the clinical decision making of patients with ACS

TABLE 2: GRACE Risk Score for UA/NSTEMI ${ }^{(18)}$

\section{Age (years) \\ $<40$ \\ $40-49$ \\ 50-59 \\ 60-69 \\ 70-79 \\ $\geq 809$ ।}

\section{Heart rate (bpm)}

$<70$

70-89

90- 109

110-149

150-199

$>200$

Systolic BP (MMHg)

$<80$

80-99

$100-119$

120-139

140-159

160-199

$>200$

\section{Creatinine $(\mathrm{mmol} / \mathrm{l})$}

0-35

35-70

$71-105$

106- 140

141-176

177-353

$>354$

Killip Class

I

II

III

IV

Cardiac Arrest at admission

Elevated cardiac marker

ST-segment deviation

Grace Risk Score:

Low

Intermediate

High

\begin{tabular}{|c|}
\hline Points \\
\hline \\
\hline \\
\hline 18 \\
\hline 36 \\
\hline 55 \\
\hline 73 \\
\hline
\end{tabular}

\begin{tabular}{|c|}
\hline \\
\hline \\
\hline 7 \\
\hline 13 \\
\hline 23 \\
\hline 36 \\
\hline 46 \\
\hline
\end{tabular}

\begin{tabular}{|c|}
\hline 63 \\
\hline 58 \\
\hline 47 \\
\hline 37 \\
\hline 26 \\
\hline 11 \\
\hline 0 \\
\hline
\end{tabular}

\begin{tabular}{|c|}
\hline 2 \\
\hline 5 \\
\hline 8 \\
\hline 11 \\
\hline 14 \\
\hline 23 \\
\hline 31 \\
\hline $109-140$ \\
\hline 21 \\
\hline 43 \\
\hline 64 \\
\hline 43 \\
\hline 15 \\
\hline 30 \\
\hline 108 \\
\hline
\end{tabular}


On the other end of the spectrum is the low risk patient group in whom an early invasive strategy may be detrimental. This group of patients is equally well served with a conservative strategy or an elective angiography / revascularization should they have high risk features on non-invasive testing.

\section{CONCLUSION}

There is sound evidence-based data that patients presenting with UA/NSTEMI, with the exception of the low risk group, will benefit from an early invasive strategy (within 4 to 72 hours of admission and stabilization with evidence-based medical therapy) with regard to reduced long-term rates of death and non-fatal MI. Trials that performed very early angiography (mean 9 hours) do not have an advantage in long-term survival as compared to trials that performed delayed angiography (mean of 39 hours). (II) A large percentage of patients who undergo angiography also get revascularized. Thus the timing of angiography may not necessarily be the most important variable and revascularization may be the more critical factor in determining longterm outcome.

An important lesson from the ICTUS trial is the importance of optimized medical treatment in the management of patients with UA/ NSTEMI. In the conservative arm of the study, $94 \%$ of patients were on statin therapy and $50 \%$ on clopidogrel at the time of discharge. Both these agents have been shown to reduce cardiac events in the management of UA/NSTEMI. It has been suggested that the neutral outcome of the ICTUS trial was related to the very high rates of optimal medical therapy in the two groups of patients. ${ }^{(19)}$

Future research needs to clarify the optimal timing of the invasive approach and to determine additional strategies for minimizing the early hazard of angiography. Research also needs to ascertain whether additional risk stratification will help in determining the best approach to an individual patient with UA/NSTEMI.
Abbreviations and Acronyms

\begin{tabular}{l|l} 
CRUSADE & $\begin{array}{l}\text { Can Rapid Risk Stratification of Unstable Angina Patients Suppress } \\
\text { Adverse Outcomes With Early Implementation of the ACC/AHA } \\
\text { Guidelines }\end{array}$ \\
\hline FRISC-2 & $\begin{array}{l}\text { Fragmin and Fast Revascularization during Instability In Coronary } \\
\text { Artery Disease }\end{array}$ \\
\hline GRACE & Global Registry of Acute Coronary Events \\
\hline ICTUS & Invasive Versus Conservative Treatment in Unstable Coronary \\
\hline ISAR-COOL & Syndromes Investigators \\
\hline TACTICS & Intracoronary Stenting With Antithrombotic Regimen Cooling Off \\
\hline MATE & Treat Angina With Aggrastat and Determine Cost of Therapy With \\
Invasive or Conservative Strategy
\end{tabular}


TREATMENT

STRATEGIES IN ACS

\section{REFERENCES:}

I. Bassand JP, Hamm CW, Ardissino D, et al. Guidelines for the diagnosis and treatment of non-ST-segment elevation acute coronary syndromes. Eur Heart J 2007; 28, $1598-1660$

2. Yusuf S, Flather M, Pogue J, et al. Variations between countries in invasive cardiac procedures and outcomes in patients with suspected unstable angina or myocardia infarction without ST elevation. Lancet 1998; 352:507-5।4.

3. Mehta SR, Cannon CP, Fox KA, Wallentein L, et al. Routine vs. Selective Invasive Strategies in Patients With Acute Coronary Syndromes. A collaborative meta-analysis of randomised trials. JAMA 2005; 293: 2908-2917.

4. De Winter RJ, Windhausen F, Cornel JH, Dunselman PH, et al. Invasive versus Conservative Treatment in Unstable coronary Syndromes (ICTUS) Investigators. N Engl J Med 2005; 353: 1095-I I 04

5. Hirsch A, Windhausen F, Tijssen JG, Verheught FW, et al. Long-term outcome after early invasive versus selective invasive treatment strategy in patients with non-ST-elevation acute coronary syndrome and elevated cardiac troponin T (the ICTUS trial: a follow-up study). Lancet 2007; 369:827-35.

6. Effects of tissue plasminogen activator and a comparison of early invasive and conservative strategies in unstable angina and non-Q-wave myocardial infarction. Results of the TIMI IIIB Trial. Thrombolysis in Myocardial Ischemia. Circulation 1994; 89: 1545-1556.

7. Boden WE, O'Rouke RA, Crawford MH, et al. Outcomes in patients with acute nonQ-wave myocardial infarction randomly assigned to an invasive as compared with a conservative management strategy.Veterans Affair Non-Q-Wave Infarction Strategies in Hospital (VANQWISH) Trial Investigators. N Eng J Med 1998; 338: I785- 1792.

8. McCullough PA, O'Neil WW, Graham M, et al. A time-to-treatment analysis in the Medicine versus Angiography in Thrombolytic Exclusion (MATE) trial. J Interv Cardiol 2001; 14: 415-22.

9. Lagervist B, Husted S, Kontny F, et al. A long-term perspective on the protective effects of an early invasive strategy in unstable coronary artery disease: two-year follow-up of the FRISC-II invasive study. J Am Coll Cardiol 2002; 40: 1902-14.

10. Hoenig MR, Doust JA, Aroney CN, Scott IA. Early invasive versus conservative strategies for unstable angina \& non-ST-elevation myocardial infarction in the stent era. Cochrane Database of Systematic Reviews 2006, Issue 3. Art. No.: CD0048I5. DOI: | 0.1 002/|465 | 858.CD0048| 5.pub2.

I I. Bavry AA, Kumbhani DJ, Rassi AN Bhatt DL,Askari AA. Benefit of Early Invasive Therapy in Acute Coronary Syndromes. A meta-analysis of contemporary randomized clinical trials. J Am Coll Cardiol 2006; 48: 1319-25.

12. Fox KA, Poole-Wilson PA, Clayton TC, et al. 5-year outcome of an interventional strategy in non-ST-segment elevation acute coronary syndrome: the British Heart Foundation RITA 3 randomized trial. Lancet 2005; 366: 9|4-20.

13. Neumann FJ, Kastrati A, Pogatsa-Murray G, et al. Evaluation of prolonged antithrombotic pre-treatment ("cooling-off" strategy) before intervention in patients with unstable coronary syndromes: a randomized controlled trial. JAMA 2003; 290: I593-9.

14. Cannon CP, Weintraub WS, Demopoulos LA, et al. TACTICS (Treat Angina with Aggrastat and Determine Cost of Therapy with an Invasive or Conservative Strategy) - Thrombolysis in Myocardial Infarction 18 Investigators. Comparison of early invasive and conservative strategies in patients with unstable coronary syndromes treated with the glycoprotein Ilb/lla inhibitor tirofiban. N Engl J Med 200 I; 344: | 879- | 887.
15. Bhatt DL, Roe MT, Peterson ED, et al. Utilization of early invasive management strategies for high-risk patients with non-ST-segment elevation acute coronary syndromes: results from the CRUSADE Quality Improvement Initiative. JAMA 2004; 292: 2096-104.

16. Anderson JL, Adams CD, Antman EM, et al. ACC/AHA 2007 guidelines for the management of patients with unstable angina/non-ST-elevation myocardial infarction executive summary: a report of the American College of Cardiology / American Heart Association Task Force on Practice Guidelines. J Am Coll Cardiol 2007; 50: 652-726.

17. Antman EM, Cohen M, Bernink PJ, et al. The TIMI risk score for unstable angina / nonST-elevation MI: A method for prognostication and therapeutic decision making. JAMA 2000; 284: 835-842.

18. Steg PG, Goldberg RJ, Gore JM, et al. Baseline characteristics, management practices, and in-hospital outcomes of patients hospitalized with acute coronary syndromes in the Global Registry of Acute Coronary Events (GRACE). Am J Cardiol 2002; 90: 358-363.

19. Boden WE. Acute coronary syndromes without ST segment elevation - What is the role of early intervention? N Engl | Med 2005; 353:| |59-6| 